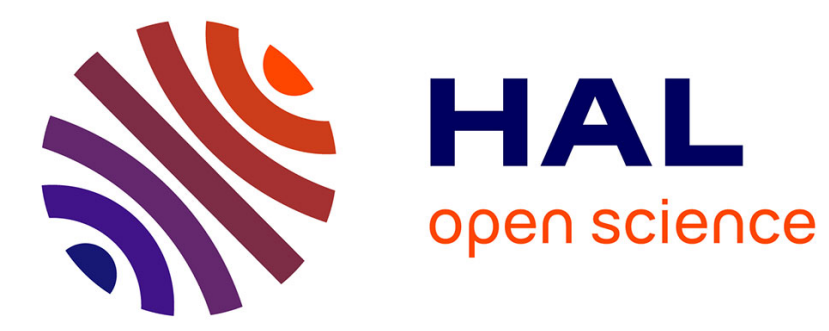

\title{
Spatial structure of first and higher harmonic internal waves from a horizontally oscillating sphere
}

\author{
Evgeny V. Ermanyuk, Jan-Bert Flór, Bruno Voisin
}

\section{To cite this version:}

Evgeny V. Ermanyuk, Jan-Bert Flór, Bruno Voisin. Spatial structure of first and higher harmonic internal waves from a horizontally oscillating sphere. Journal of Fluid Mechanics, 2011, 671, pp.364383. 10.1017/S0022112010005719 . hal-00581793v2

\section{HAL Id: hal-00581793 \\ https://hal.science/hal-00581793v2}

Submitted on 17 May 2012

HAL is a multi-disciplinary open access archive for the deposit and dissemination of scientific research documents, whether they are published or not. The documents may come from teaching and research institutions in France or abroad, or from public or private research centers.
L'archive ouverte pluridisciplinaire HAL, est destinée au dépôt et à la diffusion de documents scientifiques de niveau recherche, publiés ou non, émanant des établissements d'enseignement et de recherche français ou étrangers, des laboratoires publics ou privés. 


\title{
Spatial structure of first and higher harmonic internal waves from a horizontally oscillating sphere
}

\author{
E. V. ERMANYUK ${ }^{1} \uparrow$, J.-B. FLÓR ${ }^{2}$ AND B. VOISIN ${ }^{2}$ \\ ${ }^{1}$ Lavrentyev Institute of Hydrodynamics, Siberian Division of the Russian Academy of Science, \\ Prospekt Lavrentyev 15, Novosibirsk 630090, Russia \\ ${ }^{2}$ Laboratoire des Écoulements Géophysiques et Industriels, CNRS - Université de Grenoble, \\ BP 53, 38041 Grenoble, France
}

(Received 25 February 2010; revised 30 August 2010; accepted 30 October 2010;

first published online 10 February 2011)

An experimental study is presented on the spatial structure of the internal wave field emitted by a horizontally oscillating sphere in a uniformly stratified fluid. The limits of linear theory and the nonlinear features of the waves are considered as functions of oscillation amplitude. Fourier decomposition is applied to separate first harmonic waves at the fundamental frequency and higher harmonic waves at multiples of this frequency. For low oscillation amplitude, of $10 \%$ of the sphere radius, only the first harmonic is significant and the agreement between linear theory and experiment is excellent. As the oscillation amplitude increases up to $30 \%$ of the radius, the first harmonic becomes slightly smaller than its linear theoretical prediction and the second and third harmonics become detectable. Two distinct cases emerge depending on the ratio $\Omega$ between the oscillation frequency and the buoyancy frequency. When $\Omega>0.5$, the second harmonic is evanescent and localized near the sphere in the plane through its centre perpendicular to the direction of oscillation, while the third harmonic is negligible. When $\Omega<0.5$, the second harmonic is propagative and appears to have an amplitude that exceeds the amplitude of the first harmonic, while the third harmonic is evanescent and localized near the sphere on either side of the plane through its centre perpendicular to the direction of oscillation. Moreover, the propagative first and second harmonics have radically different horizontal radiation patterns and are of dipole and quadrupole types, respectively.

Key words: baroclinic flows, internal waves, topographic effects

\section{Introduction}

The interaction of the barotropic tidal flow with seafloor topography is known to provide a significant flux of energy into the internal tidal motion (see e.g. Morozov 1995; Vlasenko, Stashchuk \& Hutter 2005; Garrett \& Kunze 2007). This has motivated many investigations on internal tidal emission due to interaction with twodimensional topographies. Two basic types of topography have been considered: a continental slope forming the transition between a shallow shelf and a deep basin (as studied experimentally by Baines \& Fang 1985, Gostiaux \& Dauxois 2007 and Zhang, King \& Swinney 2008, and numerically by Lamb 1994, 2007, Gerkema, Staquet \&

$\dagger$ Email address for correspondence: ermanyuk@hydro.nsc.ru 
Bouruet-Aubertot 2006a, $b$ and Gayen \& Sarkar 2010), and a ridge on an otherwise even bottom (as studied experimentally by Matsuura \& Hibiya 1990, Peacock, Echeverri \& Balmforth 2008 and Echeverri et al. 2009, and numerically by Matsuura \& Hibiya 1990, Chen \& Beardsley 1995, Khatiwala 2003, Lamb 2004, 2007, Di Lorenzo, Young \& Llewellyn Smith 2006, Legg \& Huijts 2006, Legg \& Klymak 2008 and Korobov \& Lamb 2008). A common conclusion of these investigations is the importance of the slope of the topography: the internal tide has rays with a fixed slope set by the frequency, and its properties depend crucially on whether the slope of the topography is everywhere smaller than the slope of the rays, a configuration called subcritical, or locally larger, a configuration called supercritical. The internal tide is stronger in the supercritical case, owing to the existence of critical rays tangent to the topography. Available theories, however, consider topographies with slopes that are either infinitely small, corresponding to flat topography, or infinitely large, corresponding to a step or a knife-edge, as reviewed by Garrett \& Kunze (2007). Significant effort is currently devoted to building theories for finite slopes, for example by Pétrélis, Llewellyn Smith \& Young (2006), Balmforth \& Peacock (2009) and Echeverri \& Peacock (2010).

Now, at small excursion $A$ of the barotropic tide compared with the horizontal scale $\ell$ of the topography, the generation of the internal tide by the barotropic oscillation over the topography is equivalent to the generation of internal waves by the oscillation of the topography in a fluid at rest. For this latter problem, a topography exists, which has a slope varying continuously from zero to infinity and has been studied over the past five decades: the semi-circular or semi-elliptical cylinder on a plane bottom, equivalent to a circular or elliptical cylinder in an unbounded fluid, studied experimentally by Mowbray \& Rarity (1967), Makarov, Neklyudov \& Chashechkin (1990), Sutherland et al. (1999), Sutherland \& Linden (2002), Zhang, King \& Swinney (2007), Ermanyuk \& Gavrilov (2008) and Thomas, Marino \& Dalziel (2009), to name but a few. The linear theory of Hurley (1997) and Hurley \& Keady (1997) has been seen to yield excellent quantitative agreement with the measurements.

As the ratio $A / \ell$ increases, sometimes called excursion parameter for internal tides and Keulegan-Carpenter number for internal waves, nonlinear effects manifest themselves in the form of higher harmonics $n \omega$ of the fundamental oscillation frequency $\omega$, with $n=2,3, \ldots$, subject to the requirement $n \omega<N$ that the waves can propagate, with $N$ being the buoyancy frequency. Higher harmonics have been observed by Mowbray \& Rarity (1967), Makarov et al. (1990), Sutherland et al. (1999), Sutherland \& Linden (2002), Zhang et al. (2007), Ermanyuk \& Gavrilov (2008) and Thomas et al. (2009) for the cylinder, and Lamb (2004), Tabaei, Akylas \& Lamb (2005), Gerkema et al. (2006a, b), Legg \& Huijts (2006), Korobov \& Lamb (2008) and Gayen \& Sarkar (2010) for various topographies.

Two mechanisms can produce higher harmonics, as discussed e.g. by Zhang et al. (2007). For $A / \ell>1$, the dominant mechanism is advection (by the barotropic flow for a topography at rest, by the boundary forcing for an oscillating body); the theory by Bell (1975a) and Khatiwala (2003) predicts amplitudes scaling linearly as $A$ for all the harmonics. In this parameter regime, Legg \& Huijts (2006) have observed four harmonics for a Gaussian ridge, with relative amplitudes consistent with the theory. For $A / \ell<1$, the dominant mechanism is the interaction between wave beams (emanating from the topography and their reflections at the bottom, and in the case of a body, between wave beams emanating from the body); the theory by Tabaei et al. (2005) and Jiang \& Marcus (2009) predicts an amplitude scaling as $A^{n}$ for the $n$th harmonic. In this parameter regime, Sutherland et al. (1999), Sutherland \& Linden (2002) and Zhang et al. (2007) have observed a second harmonic for the cylinder, 
with an amplitude varying as $A^{2}$, for $A / \ell$ as low as 0.05 , with $\ell=a$ the radius of the cylinder.

Much less is known about three-dimensional geometries. Bell-shaped seamounts have been investigated experimentally by Zhang \& Boyer (1993) and numerically by Holloway \& Merrifield (1999) and Munroe \& Lamb (2005); the sphere has been investigated experimentally by Flynn, Onu \& Sutherland (2003) and Peacock \& Weidman (2005) and both experimentally and numerically by King, Zhang \& Swinney (2009). Quantitative measurements, in particular, have been performed for the sphere by Flynn et al. (2003) and King et al. (2009). However, the lack of an adequate theory, even for the linear case $A / \ell \ll 1$, with $\ell=a$ the radius of the sphere, has made the comparison with theory unsuccessful. For $A / \ell>1$, Bell $(1975 b)$ provided the three-dimensional generalization of the two-dimensional theory of Bell $(1975 a)$, but for $A / \ell<1$ no such generalization of the two-dimensional theory of Tabaei et al. (2005) exists, whereas the measurements for the sphere have all been performed at $A / \ell<0.6$. A second harmonic has indeed been observed by Peacock \& Weidman (2005) and King et al. (2009), but the observation was only qualitative for the former, and for the latter was limited to the frequency range $0.5<\omega / N<1$, in which the second harmonic is evanescent.

The present paper continues the investigations initiated by Voisin, Ermanyuk \& Flór (2011) of the internal wave field emitted by a horizontally oscillating sphere. Voisin et al. (2011) introduced an original linear theory for the calculation of threedimensional internal wave fields, based on Voisin (2003) and taking into account viscous effects and the unsteady effects arising from the finite time elapsed since the start-up of the motion. In addition, an original method for the measurement of internal wave motions from fluorescent dye planes (see Hopfinger et al. 1991; Flór, Ungarish \& Bush 2002) was exploited to give accurate quantitative results. The analysis focused on the linear regime and the measurements were performed in the vertical plane through the centre of the sphere along the direction of oscillation. The agreement between experiment and theory was excellent. Here the analysis focuses on the onset of nonlinearity at moderate amplitude of oscillation, applying Fourier decomposition to the experimental time series in order to separate the first to third harmonics, and on the spatial structure of these harmonics. No nonlinear theory is presented; instead, our aim is to use linear theory to identify nonlinear effects in the measurements, then to characterize these effects as fully as possible so as to set the basis for future investigators to develop a nonlinear theory.

A brief theoretical summary of the linear theory of internal wave emission by a sphere is presented in $\S 2$. The essentials of the measurement technique as well as the decomposition into harmonics are briefly considered in $\S 3$. The results are discussed in $\S 4$, separating three cases: the linear case, the nonlinear case with evanescent second harmonic and the nonlinear case with propagating second harmonic.

\section{Theory}

In an unbounded Boussinesq fluid of kinematic viscosity $v$, uniformly stratified with buoyancy frequency $N$, a sphere of radius $a$ starts at time $t=0$ to oscillate horizontally at the frequency $\omega<N$ with amplitude $A \ll a$, namely at the reduced frequency or frequency ratio $\Omega=\omega / N<1$, with Keulegan-Carpenter number or amplitude ratio $K e=A / a \ll 1$.

Internal waves are generated, which we assume of small enough amplitude for linear theory to apply. The hydrostatic assumption, which would restrict the frequency range 


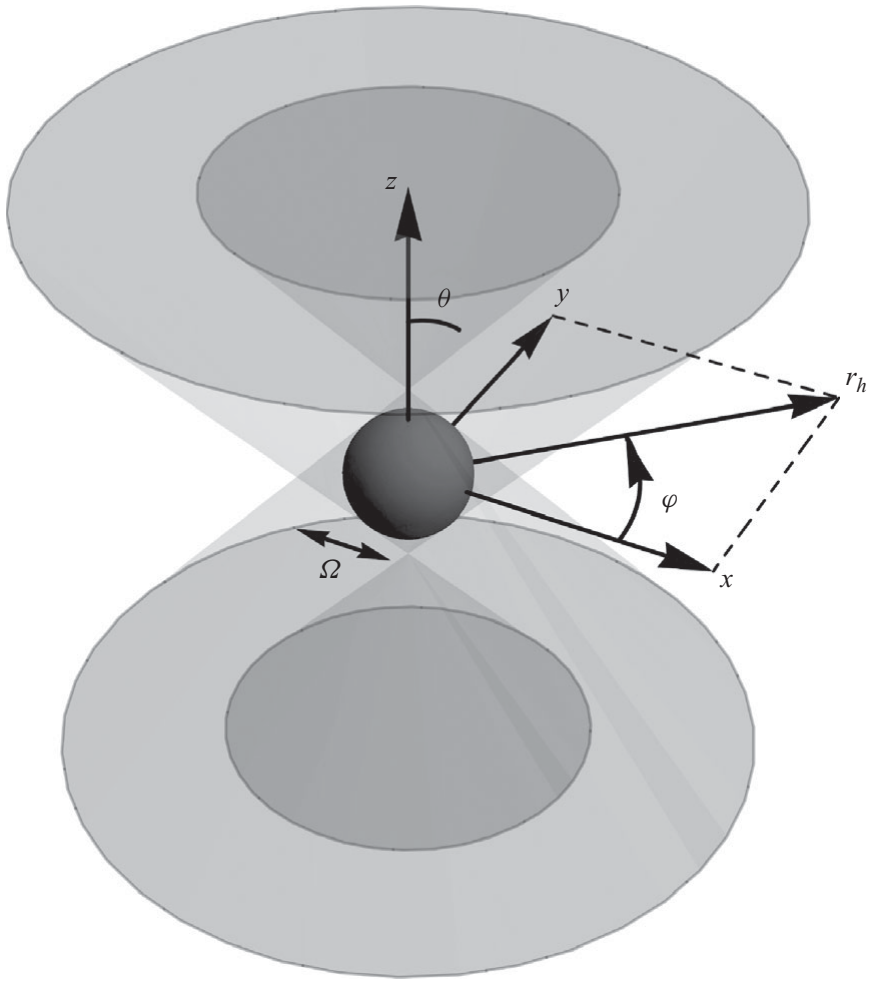

FIGURE 1. Geometry of linear internal waves from a sphere oscillating horizontally at the reduced frequency $\Omega$. The waves propagate inside a conical shell delimited by the two double cones of the vertical axis and the semi-angle $\theta=\arccos \Omega$ tangent to the sphere above and below.

to $\Omega \ll 1$, is not made. Close to the sphere, the waves are steady inviscid and propagate up and down at the angle $\theta=\arccos \Omega$ to the vertical, forming beams inside a conical shell delimited by the two double cones of vertical axis and semi-angle $\theta$ tangent to the sphere above and below. This geometry is illustrated in figure 1 in a Cartesian system of coordinates $(x, y, z)$, with origin at the centre of the sphere, vertical $z$-axis, and $x$-axis along the direction of oscillation. Away from the sphere, the beams widen and the wave profiles flatten under the joint influences of unsteadiness and viscosity. In the following, both influences are assumed small, which in terms of the product $\omega t$ and the Reynolds-Stokes number $R e=2 \omega a^{2} / v$ means $\omega t \gg 1$ and $R e \gg 1$, respectively.

On these assumptions, the waves have been calculated by Voisin et al. (2011) based on earlier work by Voisin (2003). In complex notation and cylindrical coordinates $\left(r_{h}, \varphi, z\right)$, the vertical velocity is obtained as

$$
\begin{array}{r}
W=\frac{2 \Omega^{3}}{1+B(\Omega)} \exp [-\mathrm{i}(\omega t+\Theta)] \cos \varphi \operatorname{sign} Z \int_{0}^{\Omega /(\alpha|Z|)} \exp \left(-\beta K^{3}|Z| / \Omega\right) \\
\times K j_{1}(K) \mathrm{J}_{1}\left(K R_{h} \Omega\right) \exp \left[-\mathrm{i} K|Z|\left(1-\Omega^{2}\right)^{1 / 2}\right] \mathrm{d} K,
\end{array}
$$

with $(A, 0,0) \sin (\omega t+\Theta)$ being the position of the centre of the sphere, $\mathrm{J}_{1}(x)$ being a cylindrical Bessel function and $j_{1}(x)=(\sin x) / x^{2}-(\cos x) / x$ being a spherical Bessel function. The coordinates $(x, y, z)$ and $r_{h}$ have been non-dimensionalized as $(X, Y, Z)=(x, y, z) / a$ and $R_{h}=r_{h} / a$, and the velocity components $(u, v, w)$ as 


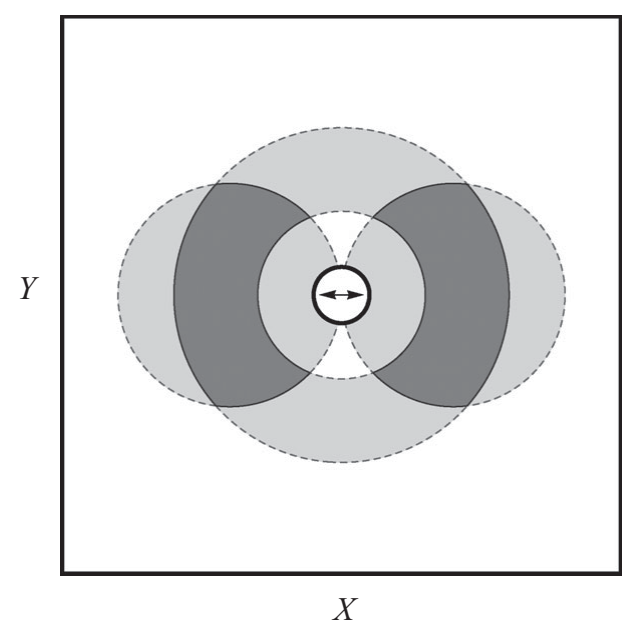

FIGURE 2. Horizontal structure of dipolar internal waves. The annular propagation of the waves, inherited from the conical geometry of figure 1, and the dipolar radiation pattern as $\cos (\varphi)$ are represented by light grey and their combination by dark grey.

$(U, V, W)=(u, v, w) /(\omega A)$. The term

$$
B(\Omega)=\Omega^{2}\left\{1-\left(1-\Omega^{2}\right)^{1 / 2}\left[\operatorname{arccosh}(1 / \Omega)+\mathrm{i} \frac{1}{2} \pi\right]\right\}
$$

represents the effect of the stratification on the added mass of the sphere, and the two parameters

$$
\alpha=\frac{1}{\omega t\left(1 / \Omega^{2}-1\right)^{1 / 2}} \quad \text { and } \quad \beta=\frac{1}{\operatorname{Re}\left(1 / \Omega^{2}-1\right)^{1 / 2}},
$$

both small, represent the respective effects of unsteadiness and viscosity. The azimuthal variation as $\cos \varphi$ follows from the dipolar nature of forcing by the oscillation of a rigid body; it implies, in the horizontal plane, radiation along the direction of oscillation and attenuation in the perpendicular direction. When this dipolar pattern is combined with the conical propagation of internal waves, a two bean-like amplitude distribution follows in the horizontal plane, illustrated in dark grey in figure 2.

\section{Experimental set-up and data processing}

The experimental set-up, sketched in figure 3, is based on the set-up used by Voisin et al. (2011) for measuring the waves in a vertical plane, modified in order to allow the spatial reconstitution of the wave field.

Experiments were conducted in a Plexiglas tank of dimensions (height $\times$ width $\times$ length) $50 \times 97 \times 97 \mathrm{~cm}^{3}$ filled to a height of $47 \mathrm{~cm}$ with a linearly salt-stratified fluid of buoyancy frequency $N=1.19 \mathrm{rad} \mathrm{s}^{-1}$ and kinematic viscosity $v=1.2 \mathrm{~mm}^{2} \mathrm{~s}^{-1}$. A sphere, attached to a pendulum of length $l=1.3 \mathrm{~m}$, was brought into oscillation at the frequency $\omega$ by a wheel rotating around an eccentric axis. The pendulum was kept against the wheel by a counterweight. The oscillation amplitude, $A$, was kept small compared to the length of the pendulum $(A / l<0.016)$, and the motion was, to a good approximation, horizontal and sinusoidal. The amplitude was varied with the vertical position of the wheel. Two spheres were used, a large one of radius $a=3.125 \mathrm{~cm}$ and 


\begin{tabular}{cccccc}
\hline Series & Sphere & $\Omega$ & $R e$ & $K e$ & $|Z|$ \\
A & Large & 0.76 & 1500 & 0.10 & $1.67,2.23,2.89$ \\
B & Small & 0.76 & 760 & $0.13,0.16,0.20,0.27$ & $1.54,2.47,3.32,4.22$ \\
C & Small & 0.42 & 420 & $0.13,0.16,0.20,0.27$ & $1.54,2.47,3.32,4.22$ \\
TABLE 1. Non-dimensional parameters for the experimental series, namely reduced frequency $\Omega$, \\
Reynolds-Stokes number Re and Keulegan-Carpenter number Ke, together with positions $|Z|$ \\
of the dye planes at which the measurements are performed.
\end{tabular}

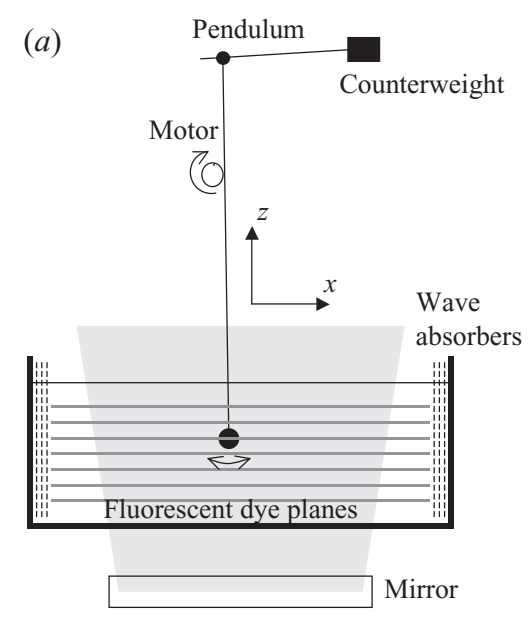

(b)

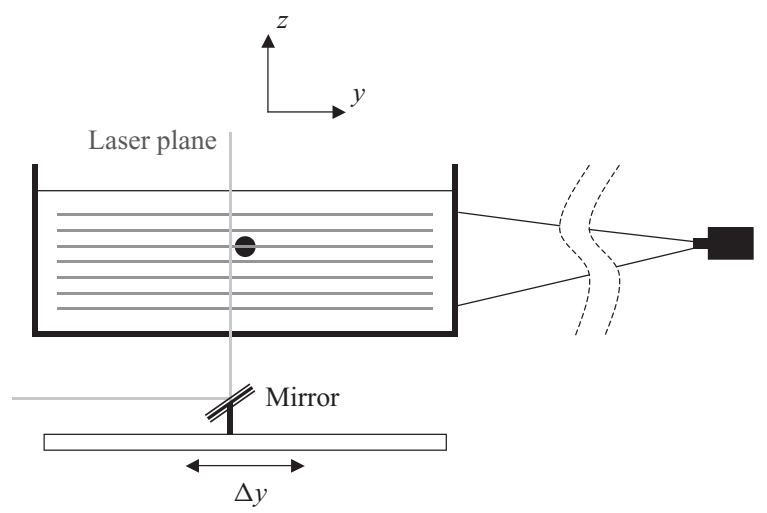

FIGURE 3. Sketch of the experimental set-up in $(a)$ front view and $(b)$ side view, with the dark grey lines denoting the fluorescein dye planes and the light grey line the laser plane. The mobility of the laser plane in the $y$-direction allows the measurement of successive planes, and the reconstitution of the horizontal wave field at levels $z / a$. These levels differ from one experiment to another.

a small one of radius $a=2.25 \mathrm{~cm}$. Three series of experiments, called A, B and C, were performed, of which the parameters are indicated in table 1.

The waves were visualized with the fluorescein-dye-line method employed by Hopfinger et al. (1991) and Flór et al. (2002), and the results processed according to the method described in detail by Voisin et al. (2011). A set of equidistant horizontal dye planes (at mutual distances of $2 \mathrm{~cm}$ ) was created in the fluid prior to the experiment and illuminated by a vertical laser sheet parallel to the direction of oscillation, so as to create an image of dye lines materializing the deformation of isopycnal lines within the fluid. The vertical displacement of the lines was determined with an accuracy of approximately 0.05 pixel, for the pictures taken in these experiments corresponding to $20 \mu \mathrm{m}$.

Successive images were taken with time increment $\Delta t=0.40 \mathrm{~s}$ for series $\mathrm{A}$ and $0.48 \mathrm{~s}$ for series $\mathrm{B}$ and $\mathrm{C}$. This increment was sufficiently small compared with the period of oscillation $T=2 \pi / \omega$ so that the number of images per period was large, between 14 and 26. Accordingly, the vertical velocity could be estimated from the dye-line displacement $\Delta \zeta(t)=\zeta(t+\Delta t)-\zeta(t)$ between two successive images, as 
$w(t)=\Delta \zeta(t) / \Delta t$. Furthermore, the velocity amplitude $|w|$ was calculated as the periodaveraged r.m.s. value of the time series multiplied by $2^{1 / 2}$, similar to the procedure introduced by Sutherland \& Linden (2002) and Flynn et al. (2003). To discriminate the effects of fundamental and multiple frequencies, Fourier filtering of the harmonics was performed, as did Zhang et al. (2007), King et al. (2009) and Thomas et al. (2009). The amplitude $\left|w_{n}\right|$ of each harmonic, with $n=1,2, \ldots$, was determined in the same way. The contribution of harmonics higher than the third was found to be negligible (of the order of the noise level), so that $|w|=\left(\left|w_{1}\right|^{2}+\left|w_{2}\right|^{2}+\left|w_{3}\right|^{2}\right)^{1 / 2}$.

The accuracy of this procedure was evaluated by generating numerically 'clean' signals with amplitudes equivalent to those observed experimentally for each harmonic. Adding random noise uniformly distributed between \pm 0.1 pixel and applying the procedure to the 'clean' and 'noisy' signals separately, the error was identified as the difference between the results for the two, with the worst combination of parameters yielding the maximum error. In this way, a 'safe' error estimate was obtained. In practice, for each series of experiments the worst combination of parameters corresponded to the lowest oscillation amplitude, i.e. the lowest $\mathrm{Ke}$.

Attention was focused on the steady state, which under typical experimental conditions is after 10 oscillation periods, as evaluated experimentally by Ermanyuk $\&$ Gavrilov $(2005,2008)$ and theoretically by Voisin et al. (2011). Measurements were taken after 20 oscillations, so that period-to-period variations of the wave amplitudes could be neglected and $\alpha$ could be set safely at zero in (2.1).

To reconstruct the three-dimensional wave field, measurements were performed in several parallel planes shifted in the transverse direction by $\Delta y=1.6 \mathrm{~cm}$, and the results were spline-interpolated for intermediate values of $y$. The parallel plane views were obtained by shifting the mirror used to reflect the laser sheet below the tank with the help of a traverse mechanism driven by a computer-controlled step motor (see figure 3). At each position, starting with $y=0$, the mirror remained fixed during one period of oscillation, allowing measurement of the time series for subsequent calculation of period-averaged r.m.s. velocity and Fourier analysis.

\section{Experimental results and discussion}

The experimental parameters are presented in table 1 . The results will be given in terms of the non-dimensional quantities $W=w /(\omega A)$ for the vertical velocity and $(X, Y, Z)=(x, y, z) / a$ for the system of coordinates, with origin at the centre of the sphere, $X$-axis along its direction of oscillation and upward vertical $Z$-axis. We distinguish three cases, with $\mathrm{A}$ the linear wave regime, $\mathrm{B}$ the nonlinear regime with evanescent second harmonic and $\mathrm{C}$ the nonlinear regime with propagating second harmonic. The linear regime is used as a reference case for the comparison with the theory of Voisin et al. (2011), recalled in § 2, which showed excellent agreement with experiment in the vertical plane $Y=0$.

\subsection{Case A: linear waves}

Case A involves the large sphere oscillating at the small amplitude $K e=0.1$. Figure 4 shows three profiles of vertical velocity amplitude along the $X$-axis in the planes $Z=-1.67,-2.23$ and -2.89 below the sphere. The contribution of all harmonics but the first is negligible, and as in Voisin et al. (2011) the agreement between experiment and theory is good. The same is true for the two-dimensional amplitude distributions in the planes, shown in figure 5. The distributions exhibit the typical two bean-like 


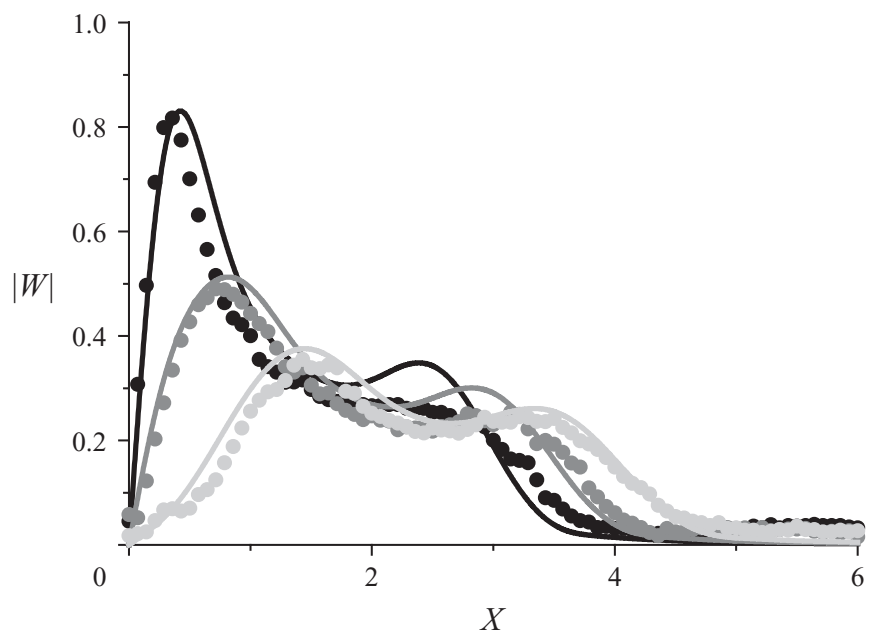

FIGURE 4. Horizontal profiles of the vertical velocity amplitude in the plane $Y=0$ for case A. The experimental profiles (dots) and the theoretical profiles (solid lines) are plotted at $Z=-1.67$ in black, -2.23 in dark grey and -2.89 in light grey.
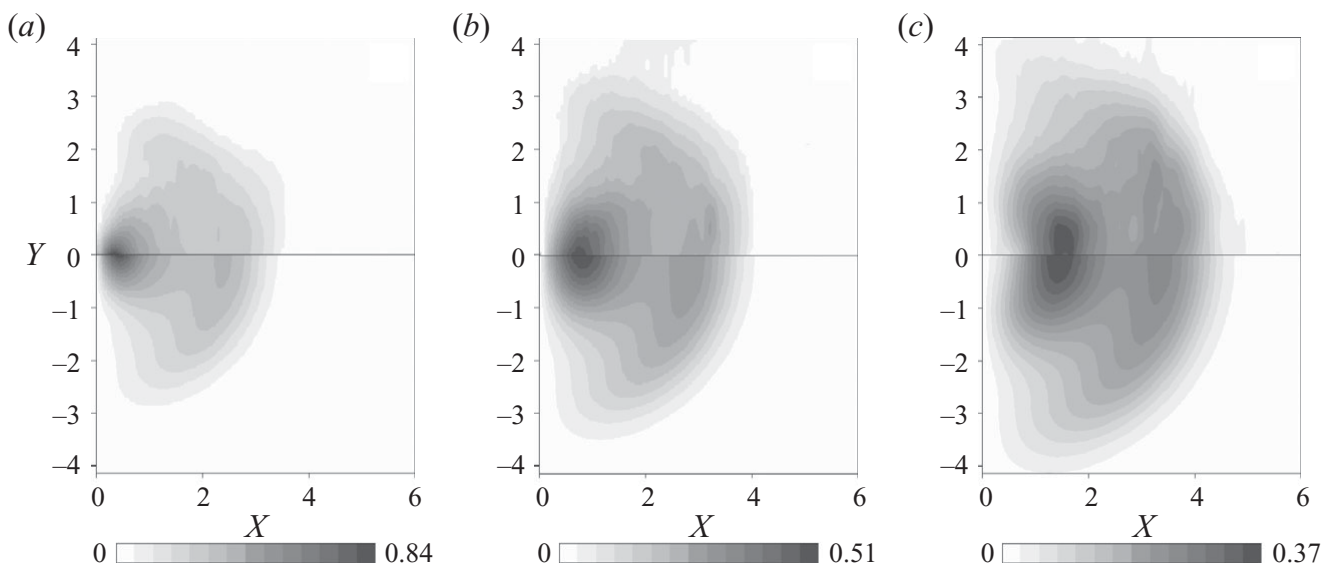

FIGURE 5. Horizontal cross-sections of the vertical velocity amplitude in case $A$ at (a) $Z=-1.67$, (b) $Z=-2.23$ and (c) $Z=-2.89$. Here the right side of the symmetry plane of figure 2 is represented, with the experimental and theoretical values, respectively, in the upper and lower halves of the images.

shape expected from figure 2. This shape is consistent with the radiation patterns observed in the numerical simulations by Holloway \& Merrifield (1999) and Munroe \& Lamb (2005) for Gaussian seamounts, exhibiting stronger amplitude along the $X$-axis parallel to the barotropic flow than along the $Y$-axis perpendicular to it.

\subsection{Case B: nonlinear waves with evanescent second harmonic at $\Omega>0.5$}

The onset of nonlinear effects due to increase of the amplitude of oscillation from $K e=0.13$ to 0.27 is represented by experimental series $\mathrm{B}$, involving the small sphere oscillating at the frequency ratio $\Omega=0.76$. At such a ratio $\Omega>0.5$, the higher 

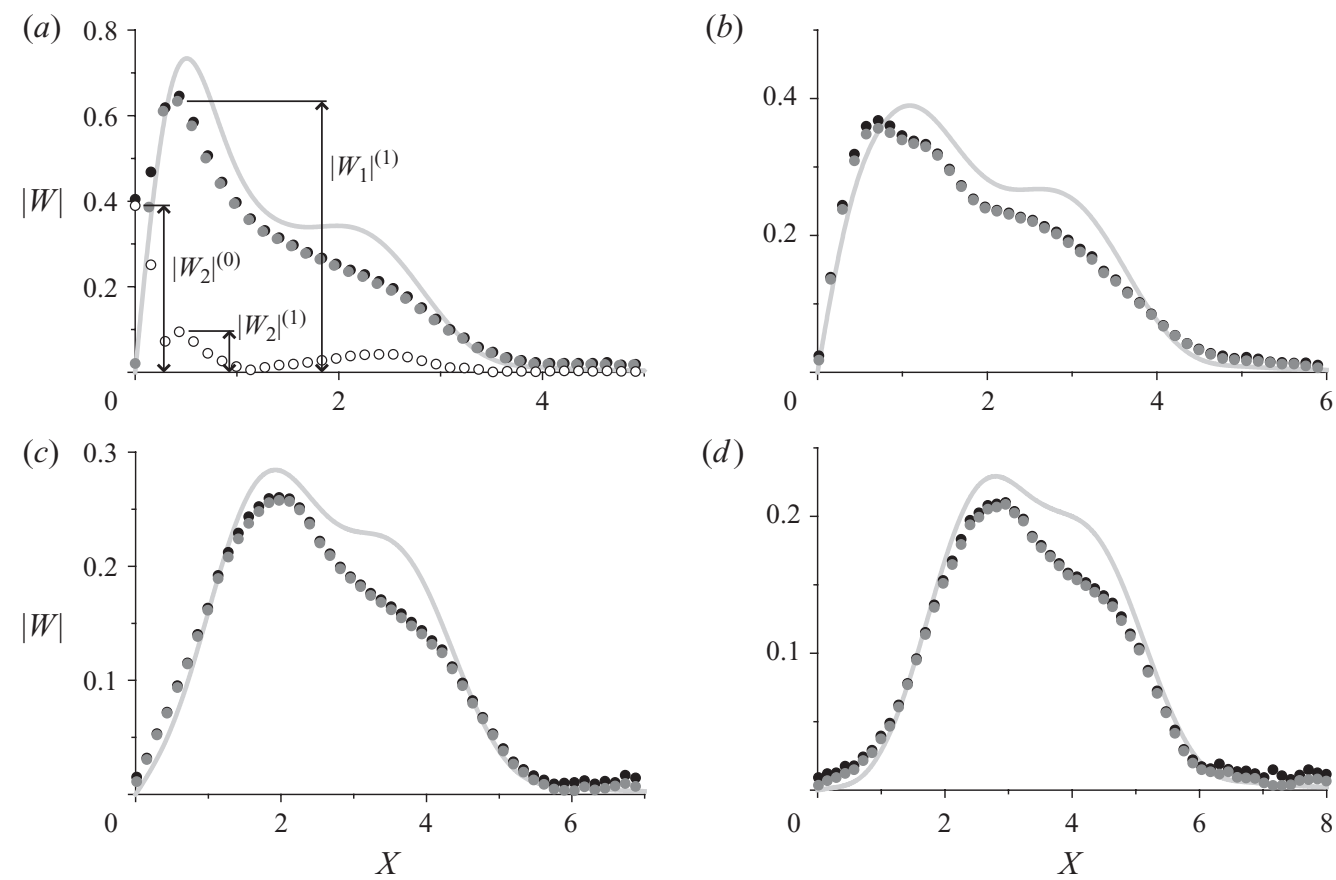

(d)

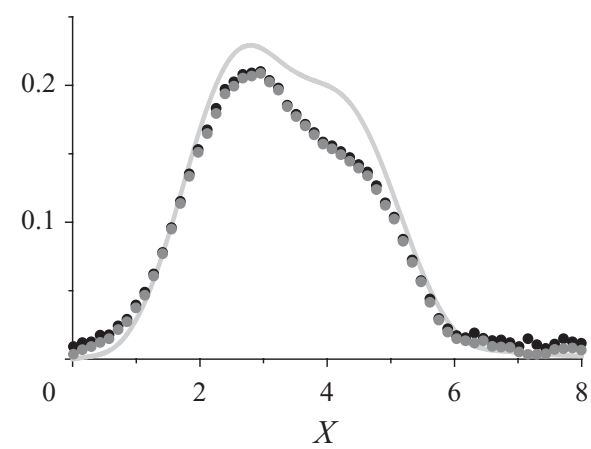

FIGURE 6. Horizontal profiles of the vertical velocity amplitude in the plane $Y=0$ for case B at $K e=0.27$ and (a) $Z=-1.54$, (b) $Z=-2.47$, (c) $Z=-3.32$ and $(d) Z=-4.22$. The black and dark grey dots and open circles represent the measured waves $|W|$ and the Fourier-filtered first and second harmonics $\left|W_{1}\right|$ and $\left|W_{2}\right|$, respectively, and the light grey lines represent the linear theory.

harmonics if present are evanescent, since their frequencies $n \omega$, with $n=2,3, \ldots$, are larger than the buoyancy frequency $N$. Accordingly, they can be observed only in the vicinity of the sphere.

This is exactly the case for the amplitude profiles in figure 6, corresponding to the largest amplitude ratio $K e=0.27$ : a second harmonic is present, which is well localized in space; namely, its Fourier-filtered profile is significant close to the sphere at $Z=-1.54$, with main maximum at $X=0$ and secondary maxima on the side, and negligible away from it at $Z=-2.47,-3.32$ and -4.22 . Higher harmonics are negligible at all $Z$.

In view of these observations, we introduce for the $n$th harmonic the notation $\left|W_{n}\right|^{(0)}$ for the maximum at $X=0$, if present, and $\left|W_{n}\right|^{(m)}$ for the successive other maxima, with increasing index $m$ as the distance of the maximum from $X=0$ increases. This notation is illustrated in figure $6(a)$ for the first and second harmonics.

The first harmonic exhibits reasonable agreement with linear theory. Its normalized peak magnitude $\left|W_{1}\right|^{(1)}$ is nearly independent of the normalized amplitude of oscillation $\mathrm{Ke}$, while the normalized peak magnitudes $\left|W_{2}\right|^{(0)}$ and $\left|W_{2}\right|^{(1)}$ of the second harmonic vary linearly with $\mathrm{Ke}$. These scalings, substantiated in figure 7 at all the positions $Z$ where the harmonics are observed, imply that the dimensional peak magnitude $\left|w_{1}\right|^{(1)}$ of the first harmonic varies linearly, as $A$, with the dimensional amplitude of oscillation $A$, and the dimensional peak magnitudes $\left|w_{2}\right|^{(0)}$ and $\left|w_{2}\right|^{(1)}$ of the second harmonic vary quadratically, as $A^{2}$. The same scalings are seen in figure 8 to apply not only to the peak magnitudes but also to the whole profiles. 

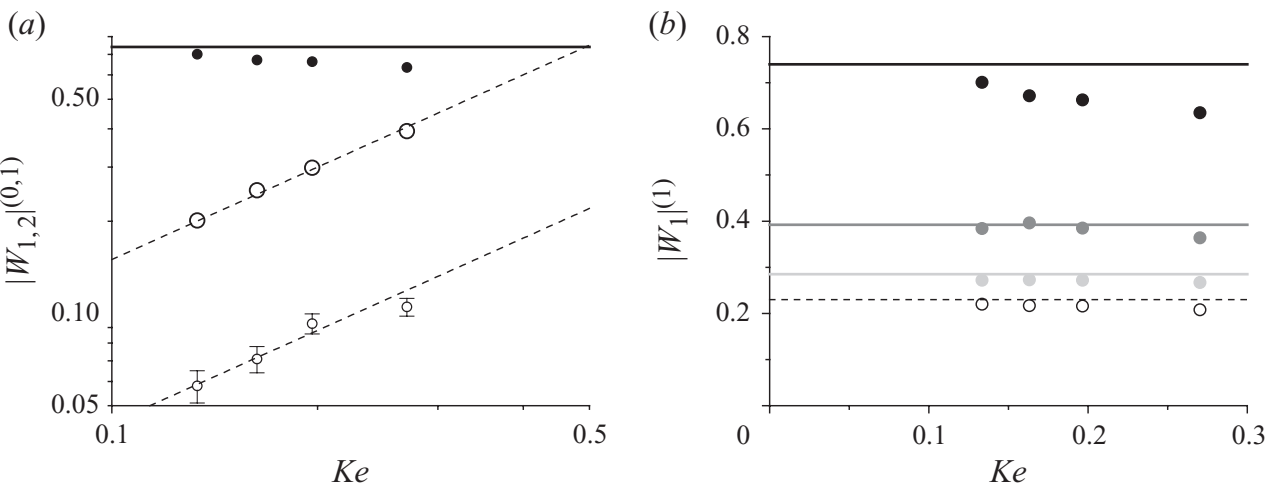

FIGURE 7. Variations of the peak magnitudes of the first and second harmonics, defined according to figure $6(a)$, with amplitude of oscillation $K e$ in case $\mathrm{B}$, for $(a)$ both harmonics at $Z=-1.54$ and $(b)$ the first harmonic only at $Z=-1.54,-2.47,-3.32$ and -4.22 . In $(a)$, the measurements of $\left|W_{1}\right|^{(1)}$ (black dots), $\left|W_{2}\right|^{(1)}$ (small open circles) and $\left|W_{2}\right|^{(0)}$ (big open circles) are compared with the theoretical prediction of $\left|W_{1}\right|^{(1)}$ (solid black line) and linear regressions for $\left|W_{2}\right|^{(1)}$ and $\left|W_{2}\right|^{(0)}$ (dashed lines). In $(b)$, the measurements of $\left|W_{1}\right|^{(1)}$ (symbols) are compared with its theoretical prediction (lines) at $Z=-1.54$ (black dots and solid line), -2.47 (dark grey dots and solid line), -3.32 (light grey dots and solid line) and -4.22 (open circles and dashed line). The error estimate, of \pm 0.006 , is represented as error bars for $\left|W_{2}\right|^{(0)}$ and is smaller than the size of the dots for $\left|W_{1}\right|^{(1)}$ and $\left|W_{2}\right|^{(1)}$.
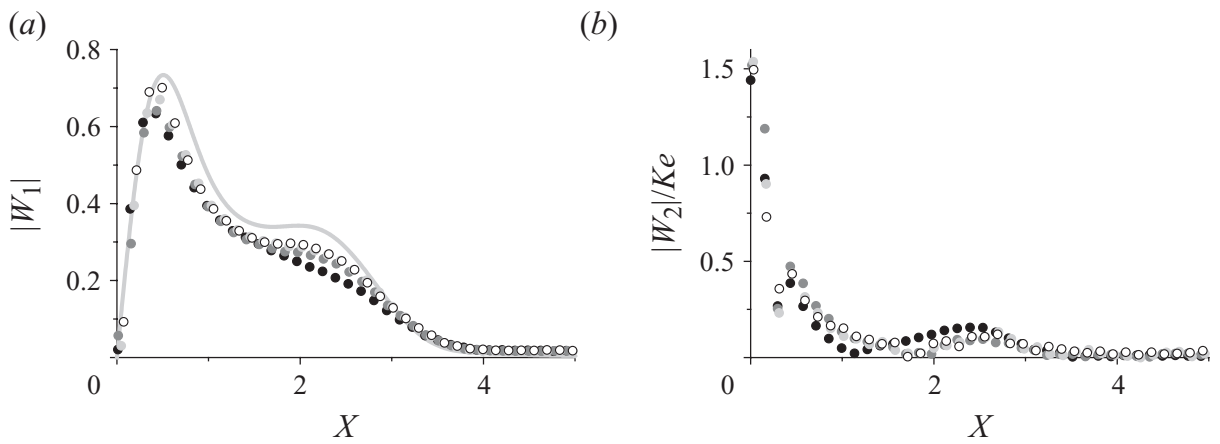

FiguRE 8. Scaled experimental profiles of $(a)$ the first harmonic and $(b)$ the second harmonic in case $\mathrm{B}$ at $Z=-1.54$ and $K e=0.13$ (open circles), 0.16 (light grey dots), 0.20 (dark grey dots) and 0.27 (black dots), together with theoretical profile of the first harmonic (light grey line).

King et al. (2009) observed in their figure 11 similar variations of the dimensional velocity as $K e$ in the plane $Y=0$, where the first harmonic is dominant, and $K e^{2}$ in the plane $X=0$, where the second harmonic is dominant. However, the variation with $\mathrm{Ke}^{2}$ does not belong to the second harmonic itself but to the zero-frequency flow accompanying it. This flow is essentially horizontal, having a vertical length scale about 20 times smaller than the horizontal length scale, and is confined to the close vicinity of the sphere. There is no such flow in the present measurements of the vertical velocity, performed at $|Z|>1.54$, that is just outside the overlap region of the wave beams defined by the wave cones tangent to the sphere above and below in figure 1. 
(a)

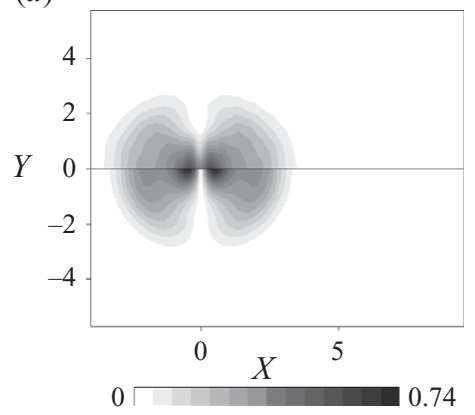

(b)

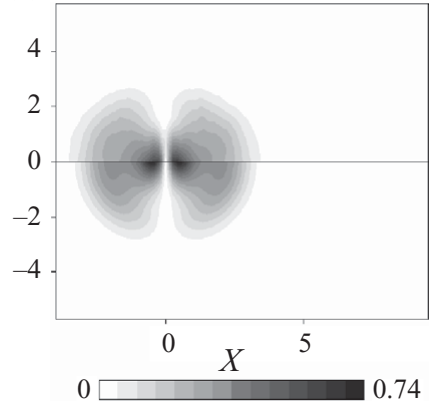

(c)

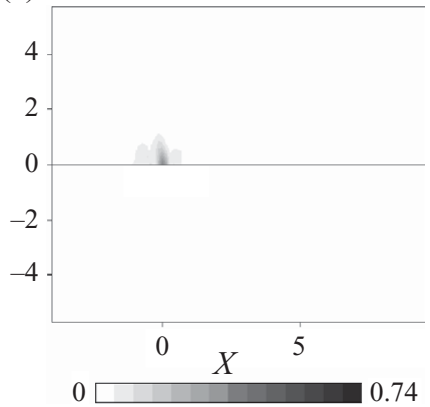

FIGURE 9. Horizontal cross-sections of the vertical velocity amplitude in case $\mathrm{B}$ at $\mathrm{Ke}=0.27$ and $Z=-1.54$ for $(a)$ the total waves $|W|$ and $(b, c)$ their harmonic components $\left|W_{1}\right|$ and $\left|W_{2}\right|$. The experimental and linear theoretical values are presented in the upper and lower halves of the images, respectively.
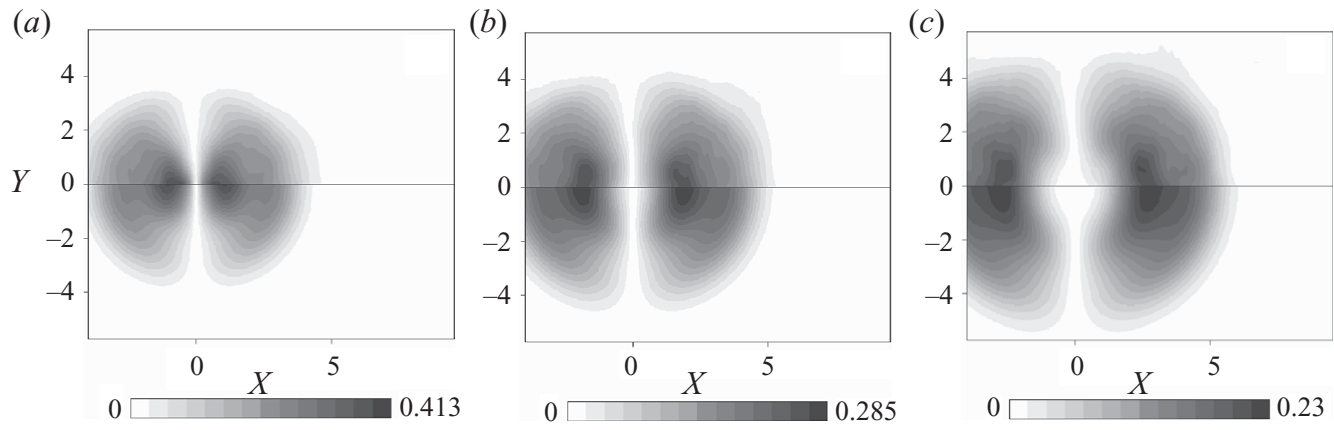

FIGURE 10. Horizontal cross-sections of the vertical velocity amplitude in case B at $\mathrm{Ke}=0.27$ and (a) $Z=-2.47$, (b) $Z=-3.32$ and (c) $Z=-4.22$. At these positions, the second harmonic component is negligible. The experimental and linear theoretical values are presented in the upper and lower halves of the images, respectively.

Also seen in figures 6-8 is a drop of the experimental values of the first harmonic below the theoretical values with $K e$. This effect, especially marked at $Z=-1.54$ and smaller at larger $|Z|$, can be attributed to the large local slopes of the isopycnal lines near the apex at $Z=-1 /\left(1-\Omega^{2}\right)^{1 / 2}=-1.54$ of the wave cone tangent to the lower part of the sphere (see figure 1): the apex corresponds to a focus of internal wave energy, so that the assumptions of linear theory are likely to be violated in its vicinity and nonlinear effects are expected to appear. As $K e$ increases further, the drop turns into saturation of the wave amplitude at all $|Z|$, as reported by Voisin et al. (2011) at $K e=0.50$ for the first harmonic and by King et al. (2009) at $K e \geqslant 0.3$ for the first harmonic and the zero-frequency flow.

The two-dimensional amplitude distribution in horizontal planes is shown at $Z=$ -1.54 in figure 9 , in which case the second harmonic is present and the first and second harmonics are plotted separately; and at $Z=-2.47,-3.32$ and -4.22 in figure 10, in which case the second harmonic is absent. The first harmonic exhibits good agreement with linear theory at all distances from the sphere; in particular, it has the same two bean-like shape as in case A. The second harmonic is confined around the transverse vertical plane $X=0$, consistent with King et al. (2009), and decreases rapidly with both $|Y|$ and $|Z|$ in this plane. 
(a)

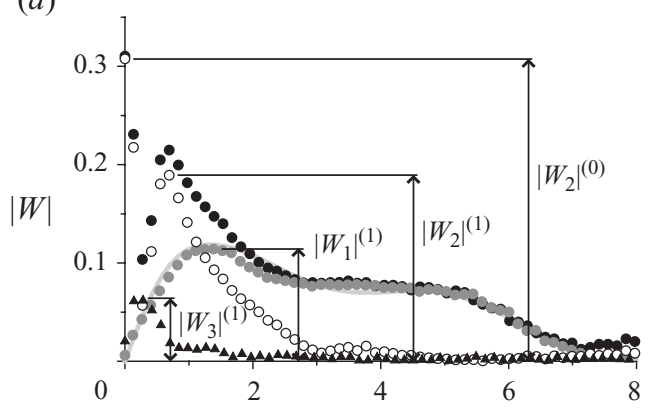

(c)

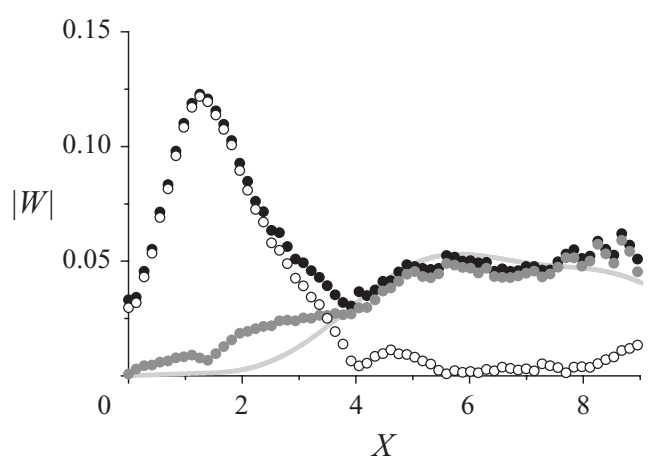

(b)

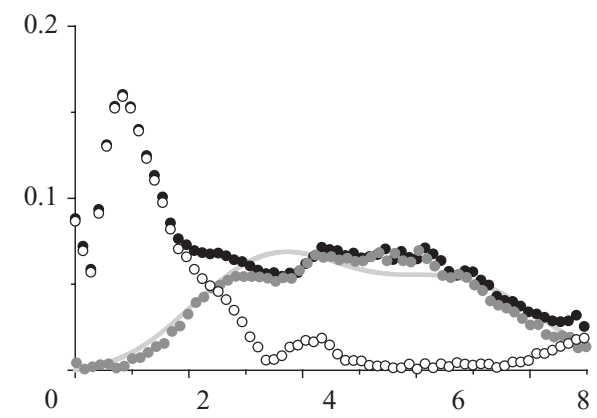

(d)

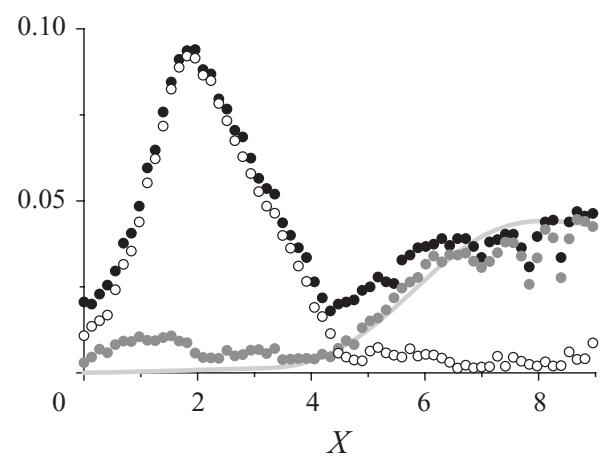

FIGURE 11. Horizontal profiles of the vertical velocity amplitude in the plane $Y=0$ for case $C$ at $K e=0.27$ and $(a) Z=-1.54,(b) Z=-2.47$, (c) $Z=-3.32$ and $(d) Z=-4.22$. The black dots, dark grey dots, open circles and black triangles represent the measured waves $|W|$ and the Fourier-filtered first, second and third harmonics $\left|W_{1}\right|,\left|W_{2}\right|$ and $\left|W_{3}\right|$, respectively, and the light grey lines represent the linear theory.

\subsection{Case $C$ : nonlinear waves with propagating second harmonic at $\Omega<0.5$}

In contrast to the cases above, experimental series $\mathrm{C}$, involving the small sphere oscillating at the frequency ratio $\Omega=0.42<0.5$, admits the radiation of a propagating second harmonic along with the first harmonic. Figure 11 shows the amplitude profiles at the same amplitude ratio $K e=0.27$ and vertical levels as in figure 6 for case B: both harmonics are observed at all levels, together with an evanescent third harmonic well localized in space and visible only at the closest level $Z=-1.54$ to the sphere, with maximum next to $X=0$.

The first harmonic exhibits good agreement with linear theory. Its normalized amplitude $\left|W_{1}\right|$ drops substantially compared with case $\mathrm{B}$, yielding a dimensional amplitude $\left|w_{1}\right|$ roughly 10 times smaller. This effect is in agreement with the dependence of the radiated wave power on frequency $\Omega$ at fixed amplitude of oscillation $\mathrm{Ke}$ : as measured experimentally by Ermanyuk (2002) and Ermanyuk \& Gavrilov (2003) and demonstrated theoretically by Voisin et al. (2011), the wave power increases from zero at $\Omega=0$ up to a maximum at $\Omega=0.85$, then decreases down to zero at $\Omega=1$. As a result, the noise-to-signal ratio increases from cases $\mathrm{B}$ to $\mathrm{C}$ as becomes evident when comparing the data in figures 6 and 11 .

The second harmonic provides an important contribution to the wave field. Its central peak $\left|W_{2}\right|^{(0)}$ is dominant at $Z=-1.54$ and secondary at $Z=-2.47$. The central peak is not observed at larger $|Z|$, while the side peak $\left|W_{2}\right|^{(1)}$ is present at all 

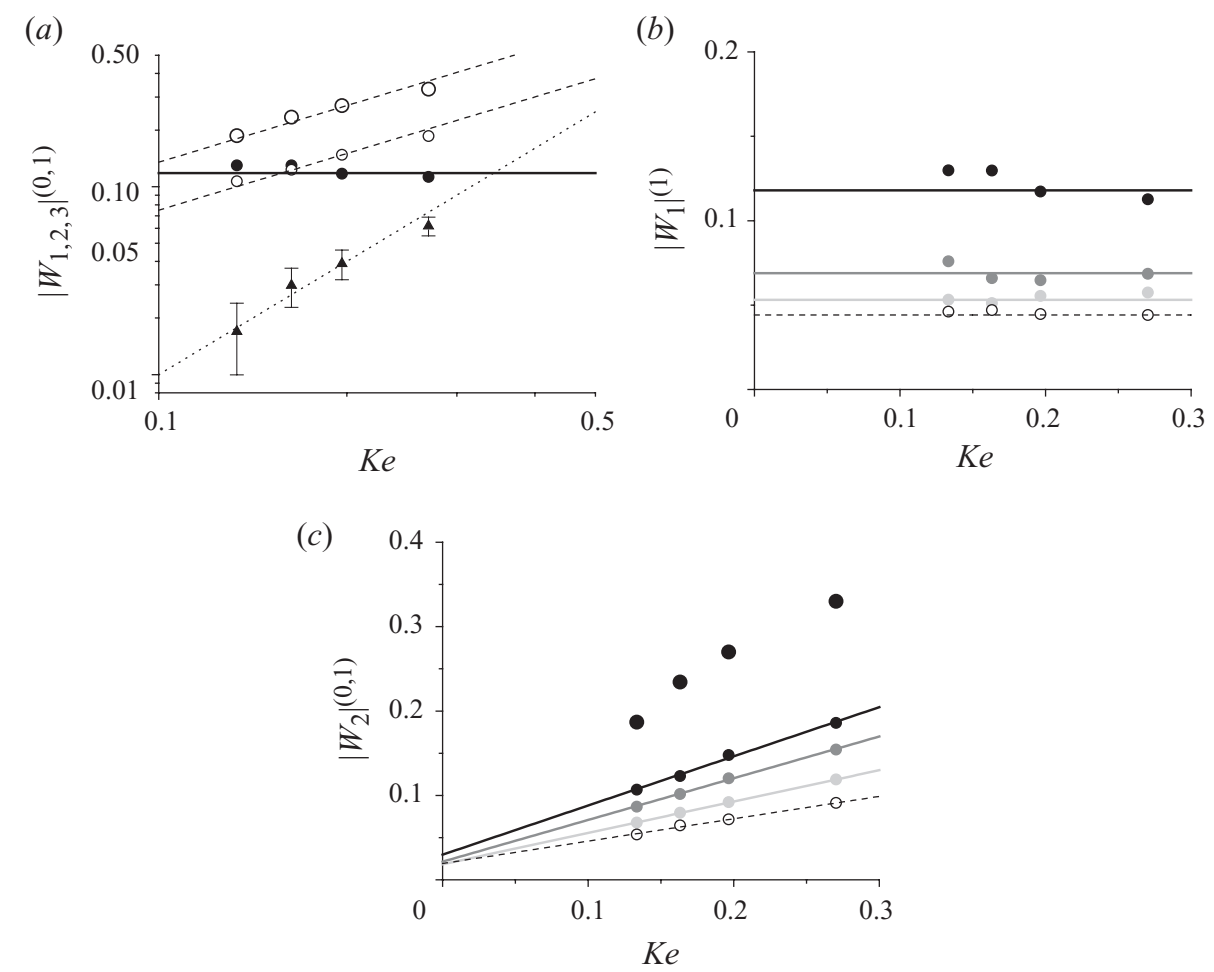

FIGURE 12. Variations of the peak magnitudes of the first to third harmonics, defined according to figure 11(a), with amplitude of oscillation $K e$ in case $\mathrm{C}$, for $(a)$ all three harmonics at $Z=-1.54$ and $(b)$ the first harmonic and $(c)$ the second harmonic at $Z=-1.54,-2.47,-3.32$ and -4.22 . In $(a)$, the measurements of $\left|W_{1}\right|^{(1)}$ (black dots), $\left|W_{2}\right|^{(1)}$ (small open circles), $\left|W_{2}\right|^{(0)}$ (big open circles) and $\left|W_{3}\right|^{(1)}$ (black triangles) are compared with the theoretical prediction of $\left|W_{1}\right|^{(1)}$ (solid black line) and linear regressions for $\left|W_{2}\right|^{(1)}$ and $\left|W_{2}\right|^{(0)}$ (dashed lines) and $\left|W_{3}\right|^{(1)}$ (dotted line). In $(b)$ and $(c)$, the measurements of $\left|W_{1}\right|^{(1)}$ and $\left|W_{2}\right|^{(1)}$ (symbols) are compared with the theoretical prediction of $\left|W_{1}\right|^{(1)}$ and a linear regression for $\left|W_{2}\right|^{(1)}$ (lines) at $Z=-1.54$ (black dots and solid lines), -2.47 (dark grey dots and solid lines), -3.32 (light grey dots and solid lines) and -4.22 (open circles and dashed lines); they are accompanied in $(c)$ by the measurements of the additional peak $\left|W_{2}\right|^{(0)}$ at $Z=-1.54$ (big black dots). The error estimate is \pm 0.006 , as in figure 7 .

$Z$. The dependence of the normalized peak magnitudes on the normalized amplitude of oscillation $\mathrm{Ke}$ is shown in figure 12 for the first to third harmonics. On log-log scale at $Z=-1.54$ in figure $12(a)$, straight lines of slopes 0,1 and 2 are obtained for the first, second and third harmonics, respectively, implying independence from $\mathrm{Ke}$ for the first and variations as $K e$ for the second and $K e^{2}$ for the third harmonics. These variations are confirmed on linear scale at all $Z$ in figures $12(b, c)$ for the first and second harmonics. As a consequence, the variations of the dimensional peak magnitudes with the dimensional amplitude of oscillation $A$ are linear, as $A$, for the first harmonic, quadratic, as $A^{2}$, for the second and cubic, as $A^{3}$, for the third harmonics.

Formally speaking, the second harmonic, though a second-order effect, can nevertheless exceed the first harmonic which is a first-order effect, in contrast to what is generally observed for a cylinder (see Sutherland \& Linden 2002; Zhang et al. 2007; Ermanyuk \& Gavrilov 2008). In figure $12(a)$, the side peak $\left|W_{2}\right|^{(1)}$ of the 

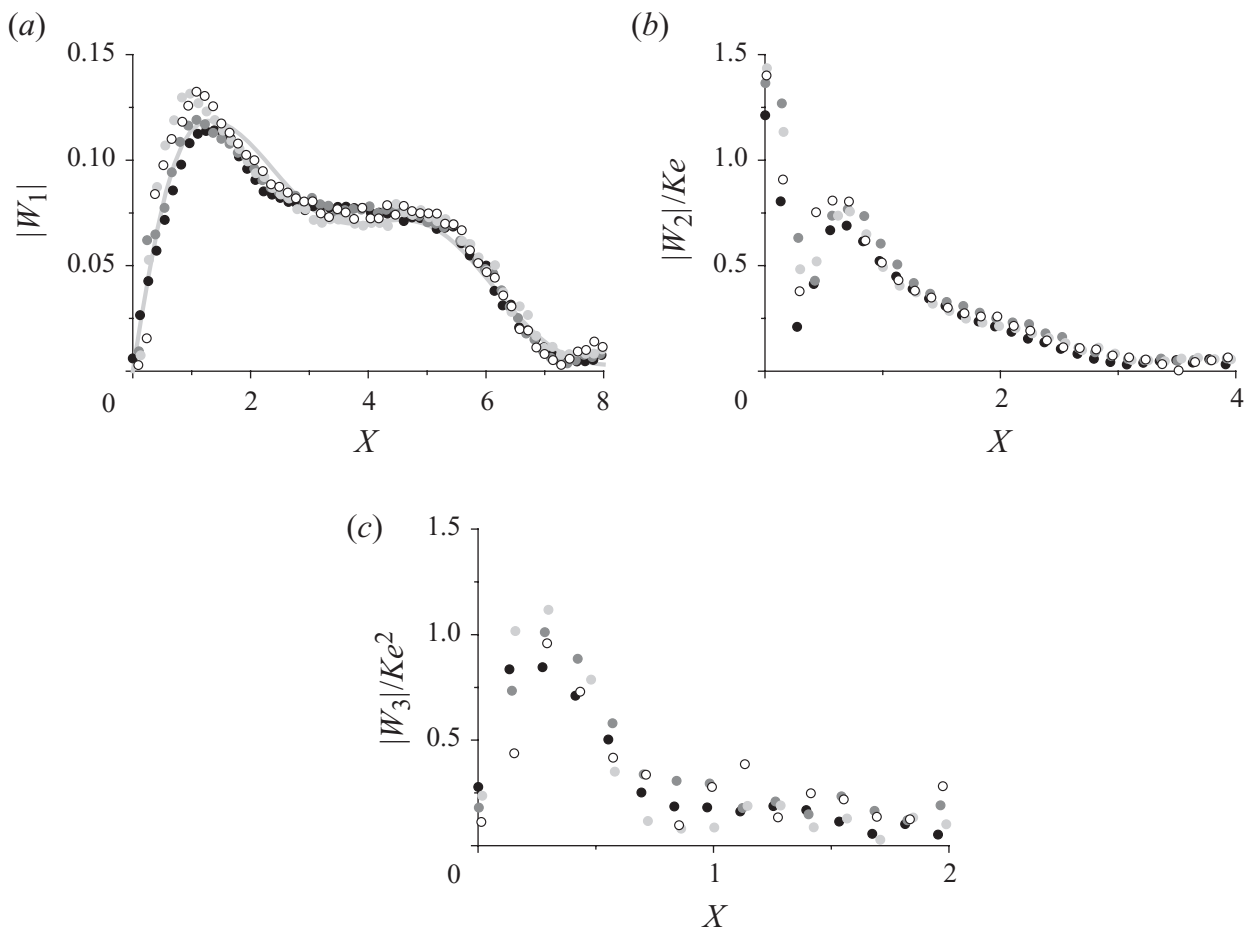

FIGURE 13. Scaled experimental profiles of $(a)$ the first harmonic, $(b)$ the second harmonic and $(c)$ the third harmonic in case $\mathrm{C}$ at $Z=-1.54$ and $K e=0.13$ (open circles), 0.16 (light grey dots), 0.20 (dark grey dots) and 0.27 (black dots), together with theoretical profile of the first harmonic (light grey line).

second harmonic is seen to overtake $\left|W_{1}\right|^{(1)}$ of the first harmonic around $K e=0.15$, a value to be compared with $K e=0.09$, at which the zero-frequency flow takes over the first harmonic in figure 11 of King et al. (2009). At $Z=-1.54$, the central peak $\left|W_{2}\right|^{(0)}$ becomes so strong with increasing $K e$ that a small departure from linearity is observed after $\mathrm{Ke}=0.2$ in figure $12(\mathrm{c})$. This behaviour indicates a tendency towards saturation of the second harmonic close to the apices of the wave cones, similar to the tendency observed for the first harmonic in case B. It may be expected that the tendency towards saturation is sensitive to the value of the Reynolds number. The existing body of data does not allow us to draw a final conclusion concerning this issue, which represents an area for future research.

The small non-zero value at $K e=0$ in figure $12(c)$ obtained from the linear regressions characterizes the overall level of noise in the experiments. Plotting each harmonic scaled by the appropriate power of the amplitude of oscillation in figure 13 reveals again that the scalings are confirmed for the entire profiles (the increase in noise level with harmonic order is an artefact due to the smaller number of experimental images per period, from 26 for the first harmonic to 13 for the second to 9 for the third).

The two-dimensional structure of the wave field in horizontal planes is visualized in figures 14 and 15 . The variations of the amplitude with azimuthal angle $\varphi$ are seen to be different for the first harmonic, propagating at the angle $\theta=\arccos \Omega=65^{\circ}$ to the vertical, and the second harmonic, propagating at the smaller angle $\arccos (2 \Omega)=33^{\circ}$, hence observed at smaller $|X|$ and $|Y|$. Fourier decomposition allows the separation of 
(a)

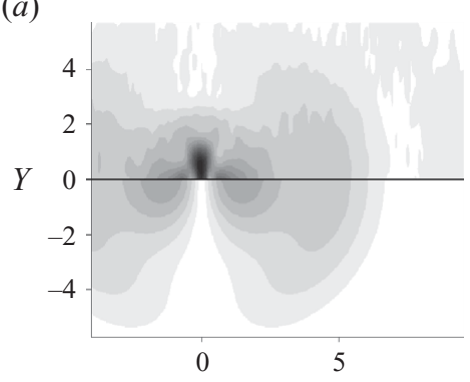

(c)

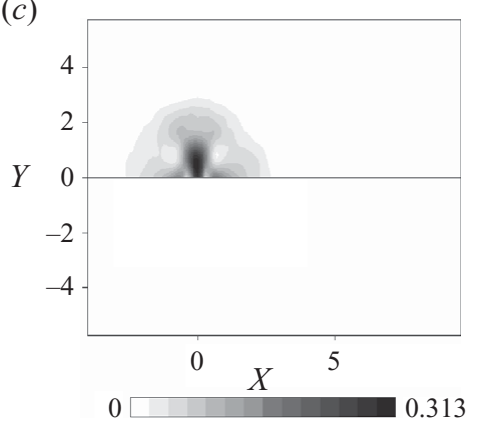

(b)

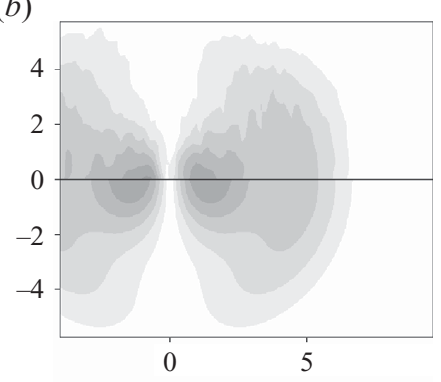

(d)

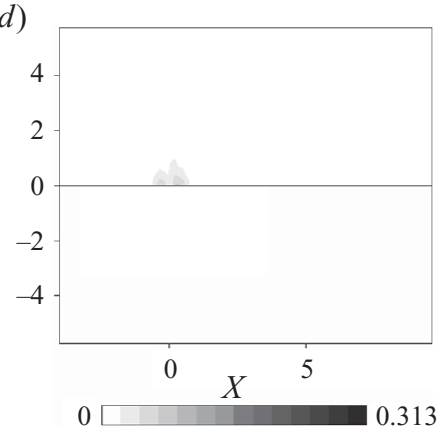

FIgURE 14. Horizontal cross-sections of the vertical velocity amplitude in case $\mathrm{C}$ at $K e=0.27$ and $Z=-1.54$ for $(a)$ the total waves $|W|$ and $(b-d)$ their harmonic components $\left|W_{1}\right|$ to $\left|W_{3}\right|$. The mode of representation is the same as in figure 9.

the two in the regions where they overlap. In spite of appreciable noise-to-signal ratio, even at moderate $\mathrm{Ke}$, the measured and calculated first harmonics are in reasonably good agreement and exhibit the same two bean-like shape as observed in cases A and $\mathrm{B}$, associated with dipolar azimuthal variations of the form $\cos \varphi$, as illustrated in figure 2. The second harmonic exhibits a different clover-like shape, which may be associated with quadrupolar azimuthal variations of the form $\cos (2 \varphi)$, as illustrated in figure 16.

The origin of quadrupolar radiation in this context is unclear. Since the amplitude variations of the second and third harmonics are proportional to, respectively, the second and third powers of the amplitude of oscillation (as discussed in $\S 1$ ), the generation by advection is ruled out, implying the generation by nonlinear interaction. Higher harmonics have only been considered theoretically for the two-dimensional case (see Tabaei et al. 2005; Jiang \& Marcus 2009). If we look closely at the visualizations of propagating higher harmonics in the literature, all two-dimensional (see Sutherland \& Linden 2002, Zhang et al. 2007 and Thomas et al. 2009 for a cylinder, and Lamb 2004, Tabaei et al. 2005, Gerkema et al. 2006a,b and Korobov \& Lamb 2008 for various topographies), we note that the generation of higher harmonics is a very local process, taking place at the locations where the critical wave rays tangent to the body (or topography) either (i) intersect each other (or their reflection at the bottom) or (ii) touch the body (or topography). Location (i) suggests nonlinear interaction of wave beams and corresponds to locations where the linear theory for a spherical body of Voisin et al. (2011) predicts higher-order steady inviscid singularities, i.e. the apices of the wave cones tangent to the sphere at $|Z|=1 /\left(1-\Omega^{2}\right)^{1 / 2}$, and the circular intersection of these cones at $R_{h}=1 / \Omega$ in the 


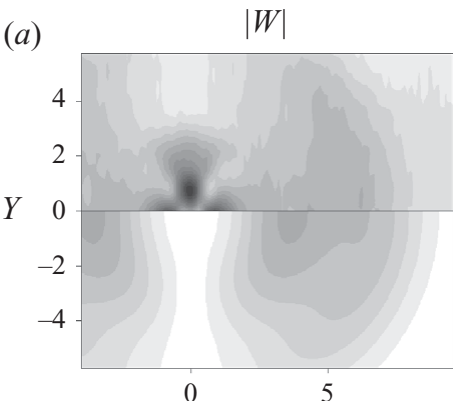

$$
0
$$

0.173
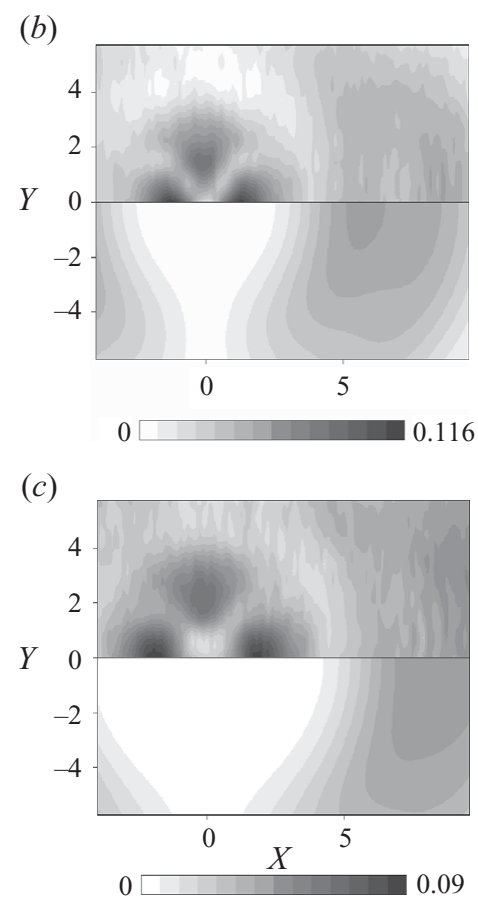
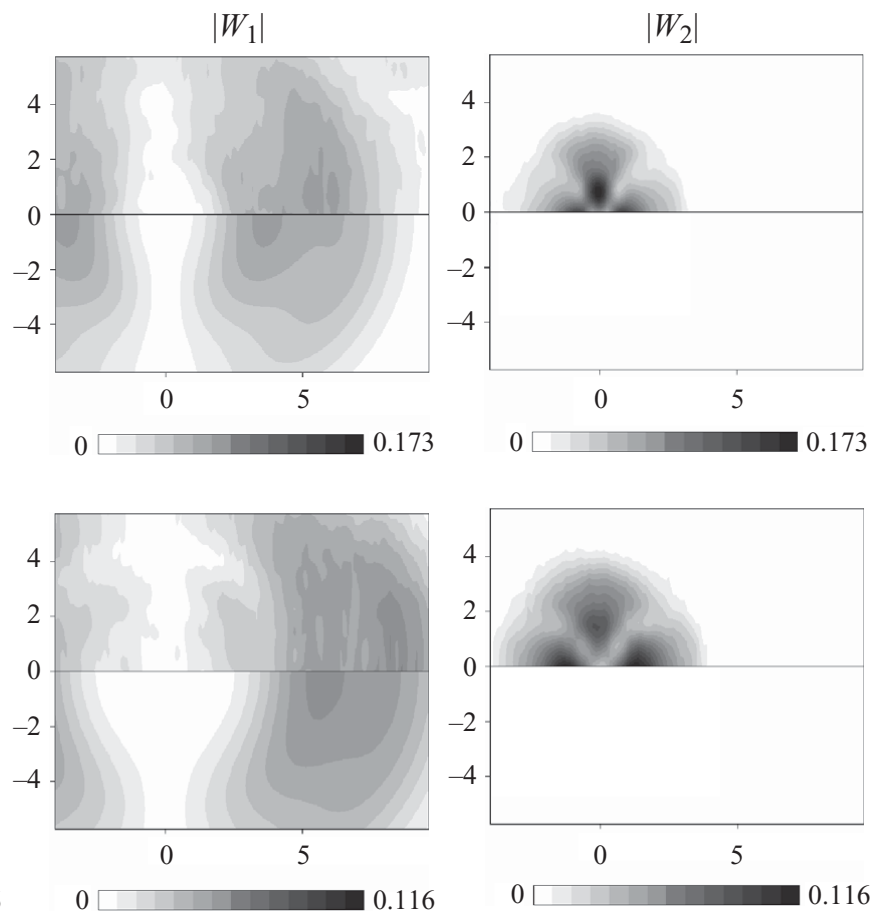

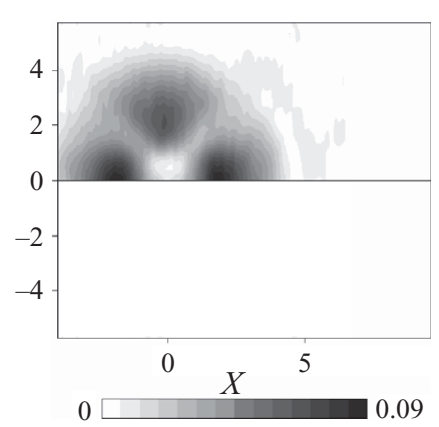

FiguRE 15. Horizontal cross-sections of the vertical velocity amplitude in case $\mathrm{C}$ at $K e=0.27$ and (a) $Z=-2.47,(b) Z=-3.32$ and (c) $Z=-4.22$ for the total wave field $|W|$ and its first and second harmonic components $\left|W_{1}\right|$ and $\left|W_{2}\right|$. The mode of representation is the same as in figures 9 and 10 .

midplane $Z=0$. Location (ii) suggests nonlinear interaction between a wave beam and the oscillatory boundary layer at the body (or topography). The boundary layer is neglected by Voisin et al. (2011).

Based on symmetry considerations alone, variations of the form $\cos (2 \varphi)$ are consistent with the observation of Ermanyuk \& Gavrilov (2008) that, in the twodimensional case of a cylinder in their figure $12(b)$, the first harmonic is antisymmetric with respect to the vertical axis $X=0$ while the second harmonic is symmetric. When combined with symmetry with respect to the vertical plane $Y=0$ in the threedimensional case of a sphere, dipolar variations of the form $\cos \varphi$ are anticipated naturally for the first harmonic and quadrupolar variations of the form $\cos (2 \varphi)$ for the second harmonic. 


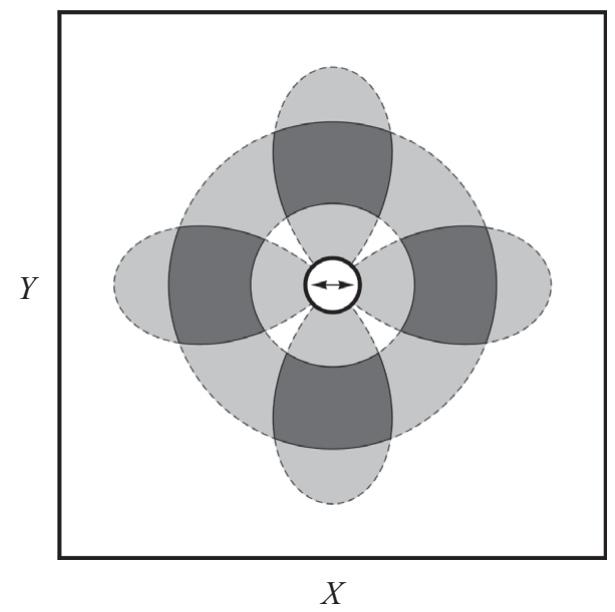

FIGURE 16. Horizontal structure of quadrupolar internal waves. The annular propagation of the waves and the quadrupolar radiation pattern as $\cos (2 \varphi)$ are represented by light grey and their combination by dark grey.

Finally, we also verify in figure 14 that the evanescent third harmonic is, as the evanescent second harmonic in case B, confined around the transverse vertical plane $X=0$ and decreases rapidly with both $|Y|$ and $|Z|$ in this plane.

\section{Conclusion}

In this paper, we have further explored the dye-line technique developed by Voisin et al. (2011) for the quantitative measurement of the spatial distribution of vertical velocities in the internal wave field emitted by a horizontally oscillating sphere. Following the approach of Sutherland \& Linden (2002) and Flynn et al. (2003), the velocity amplitudes were estimated from the period-averaged r.m.s. velocities multiplied by $2^{1 / 2}$; following the approach of Zhang et al. (2007), King et al. (2009) and Thomas et al. (2009), the velocity fluctuations were decomposed into harmonics of the oscillation frequency using Fourier analysis. Three cases were considered, as characterized in table 1.

For the linear case $\mathrm{A}$, corresponding to low ratio $\mathrm{Ke}=0.1$ of the amplitude of oscillation to the radius of the sphere, good agreement was observed between the experimental data and the linear theory presented by Voisin et al. (2011). The wave structure was dipolar and two bean-shaped in the horizontal plane, most radiation taking place along the direction of oscillation. The data exhibited high accuracy and a low level of noise, and the contribution of all harmonics but the first (at the fundamental frequency) was negligible.

For case $\mathrm{B}$, corresponding to higher amplitude ratio $\mathrm{Ke}$ up to 0.3 and a ratio $\Omega>0.5$ of the oscillation frequency to the buoyancy frequency, an evanescent second harmonic was observed near the sphere, confined around the transverse vertical plane through its centre, and was negligible away from the sphere. The amplitude of the first harmonic, which in a linear scenario should vary linearly with the amplitude of oscillation, was seen to fall gradually below this variation as $\mathrm{Ke}$ increases. This weak but detectable effect was accentuated close to the sphere, a likely consequence of the large local slopes of the isopycnal surfaces near the vertices of the wave cones, offering favourable conditions for the development of nonlinear effects leading to 
saturation. The amplitude of the second harmonic is proportional to the oscillation amplitude squared, which rules out generation by advection and suggests nonlinear wave-wave or wave-boundary-layer interaction.

For case $\mathrm{C}$, corresponding to the same amplitude ratio $\mathrm{Ke}$ as for case $\mathrm{B}$ and a frequency ratio $\Omega<0.5$, the second harmonic was seen to propagate away from the sphere and to have a quadrupolar radiation pattern, associated with a clover-shaped structure in the horizontal plane, most radiation taking place along and perpendicular to the direction of oscillation. In addition, an evanescent third harmonic was observed near the sphere, confined around the transverse vertical plane through its centre. The most striking observation is that, in contrast to its two-dimensional counterpart for the cylinder, the second harmonic can exceed the first even at moderate oscillation amplitude (say, $K e>0.2$ ). The amplitude of the third harmonic is proportional to the oscillation amplitude to the third power, suggesting nonlinear interaction of the second harmonic with the first or with the oscillatory boundary layer at the sphere.

The authors gratefully acknowledge the technical help of P. Carecchio and J. Virone, and the help with image acquisition and data transfer of $\mathrm{H}$. Scolan. The referees are thanked for insightful criticisms and suggestions. This work was accomplished within the French-Russian 'Regular and Chaotic Hydrodynamics' European Research Network. E.V.E. gratefully acknowledges his appointment as a visiting professor at University Joseph Fourier from February-April 2009, during which the experiments were performed at LEGI. E.V.E. has been partially supported by RFBR project no. 09-01-00427 and by project 17.4 of the Presidium of RAS. J.B.F. acknowledges funding by grant FLOWing of the French Research Agency, and B.V. by grants PIWO of the French Research Agency and LEFE/IDAO of INSU.

\section{REFERENCES}

BAINes, P. G. \& FAnG, X.-H. 1985 Internal tide generation at a continental shelf/slope junction: a comparison between theory and a laboratory experiment. Dyn. Atmos. Oceans 9, 297-314.

Balmforth, N. J. \& Peacock, T. 2009 Tidal conversion by supercritical topography. J. Phys. Oceanogr. 39, 1965-1974.

BeLl, T. H. 1975a Lee waves in stratified flows with simple harmonic time dependence. J. Fluid Mech. 67, 705-722.

BeLL, T. H. $1975 b$ Topographically generated internal waves in the open ocean. J. Geophys. Res. 80, 320-327.

Chen, C. \& Beardssey, R. C. 1995 A numerical study of stratified tidal rectification over finiteamplitude banks. Part I. Symmetric banks. J. Phys. Oceanogr. 25, 2090-2110.

Di Lorenzo, E., Young, W. R. \& Llewellyn Smith, S. 2006 Numerical and analytical estimates of $M_{2}$ tidal conversion at steep oceanic ridges. J. Phys. Oceanogr. 36, 1072-1084.

Echeverri, P., Flynn, M. R., Winters, K. B. \& Peacock, T. 2009 Low-mode internal tide generation by topography: an experimental and numerical investigation. J. Fluid Mech. 636, 91-108.

ECHEVERRI, P. \& PeACOCK, T. 2010 Internal tide generation by arbitrary two-dimensional topography. J. Fluid Mech. 659, 247-266.

ERManyuk, E. V. 2002 The rule of affine similitude for the force coefficients of a body oscillating in a uniformly stratified fluid. Exp. Fluids 32, 242-251.

Ermanyuk, E. V. \& GaVRilov, N. V. 2003 Force on a body in a continuously stratified fluid. Part 2. Sphere. J. Fluid Mech. 494, 33-50.

ErmanyuK, E. V. \& Gavrilov, N. V. 2005 Duration of transient processes in the formation of internal-wave beams. Dokl. Phys. 50, 548-550.

ErmanyuK, E. V. \& GaVRiLOv, N. V. 2008 On internal waves generated by large-amplitude circular and rectilinear oscillations of a circular cylinder in a uniformly stratified fluid. J. Fluid Mech. 613, 329-356. 
FlóR, J.-B., Ungarish, M. \& Bush, J. W. M. 2002 Spin-up from rest in a stratified fluid: boundary flows. J. Fluid Mech. 472, 51-82.

FlynN, M. R., Onu, K. \& Sutherland, B. R. 2003 Internal wave excitation by a vertically oscillating sphere. J. Fluid Mech. 494, 65-93.

Garrett, C. \& Kunze, E. 2007 Internal tide generation in the deep ocean. Annu. Rev. Fluid Mech. 39, 57-87.

GaYen, B. \& SARKar, S. 2010 Turbulence during the generation of internal tide on a critical slope. Phys. Rev. Lett. 104, 218502.

Gerkema, T., Staquet, C. \& Bouruet-Aubertot, P. $2006 a$ Non-linear effects in internal-tide beams, and mixing. Ocean Modell. 12, 302-318.

Gerkema, T., Staquet, C. \& Bouruet-Aubertot, P. $2006 b$ Decay of semi-diurnal internal-tide beams due to subharmonic resonance. Geophys. Res. Lett. 33, L08604.

Gostiaux, L. \& Dauxois, T. 2007 Laboratory experiments on the generation of internal tidal beams over steep slopes. Phys. Fluids 19, 028102.

Holloway, P. E. \& Merrifield, M. A. 1999 Internal tide generation by seamounts, ridges, and islands. J. Geophys. Res. 104, 25937-25951.

Hopfinger, E. J., Flór, J.-B., Chomaz, J.-M. \& Bonneton, P. 1991 Internal waves generated by a moving sphere and its wake in a stratified fluid. Exp. Fluids 11, 255-261.

Hurley, D. G. 1997 The generation of internal waves by vibrating elliptic cylinders. Part 1. Inviscid solution. J. Fluid Mech. 351, 105-118.

Hurley, D. G. \& Keady, G. 1997 The generation of internal waves by vibrating elliptic cylinders. Part 2. Approximate viscous solution. J. Fluid Mech. 351, 119-138.

JiANG, C.-H. \& MARCUS, P. S. 2009 Selection rules for the nonlinear interaction of internal gravity waves. Phys. Rev. Lett. 102, 124502.

Khatiwala, S. 2003 Generation of internal tides in an ocean of finite depth: analytical and numerical calculations. Deep-Sea Res. I 50, 3-21.

King, B., Zhang, H. P. \& Swinney, H. L. 2009 Tidal flow over three-dimensional topography in a stratified fluid. Phys. Fluids 21, 116601.

Kововоv, A. S. \& LAmb, K. G. 2008 Interharmonics in internal gravity waves generated by tide-topography interaction. J. Fluid Mech. 611, 61-95.

LAMB, K. G. 1994 Numerical experiments of internal wave generation by strong tidal flow across a finite amplitude bank edge. J. Geophys. Res. 99, 843-864.

LAMB, K. G. 2004 Nonlinear interaction among internal wave beams generated by tidal flow over supercritical topography. Geophys. Res. Lett. 31, L09313.

LAMB, K. G. 2007 Energy and pseudoenergy flux in the internal wave field generated by tidal flow over topography. Cont. Shelf Res. 27, 1208-1232.

LegG, S. \& HuiJTs, K. M. H. 2006 Preliminary simulations of internal waves and mixing generated by finite amplitude tidal flow over isolated topography. Deep-Sea Res. II 53, 140-156.

LEGG, S. \& KLYMAK, J. 2008 Internal hydraulic jumps and overturning generated by tidal flow over a tall steep ridge. J. Phys. Oceanogr. 38, 1949-1964.

Makarov, S. A., Neklyudov, V. I. \& Chashechin, Yu. D. 1990 Spatial structure of twodimensional monochromatic internal-wave beams in an exponentially stratified liquid. Izv. Atmos. Ocean. Phys. 26, 548-554.

MatsuURA, T. \& HibiYa, T. 1990 An experimental and numerical study of the internal wave generation by tide-topography interaction. J. Phys. Oceanogr. 20, 506-521.

Morozov, E. G. 1995 Semidiurnal internal wave global field. Deep-Sea Res. I 42, 135-148.

MowbraY, D. E. \& Rarity, B. S. H. 1967 A theoretical and experimental investigation of the phase configuration of internal waves of small amplitude in a density stratified liquid. J. Fluid Mech. 28, $1-16$.

Munroe, J. R. \& LAmb, K. G. 2005 Topographic amplitude dependence of internal wave generation by tidal forcing over idealized three-dimensional topography. J. Geophys. Res. 110, C02001.

Peacock, T., Echeverri, P. \& Balmforth, N. J. 2008 An experimental investigation of internal tide generation by two-dimensional topography. J. Phys. Oceanogr. 38, 235-242.

Peacock, T. \& Weidman, P. 2005 The effect of rotation on conical wave beams in a stratified fluid. Exp. Fluids 39, 32-37.

Pétrélis, F., Llewellyn Smith, S. \& Young, W. R. 2006 Tidal conversion at a submarine ridge. J. Phys. Oceanogr. 36, 1053-1071. 
Sutherland, B. R., Dalziel, S. B., Hughes, G. O. \& Linden, P. F. 1999 Visualization and measurement of internal waves by 'synthetic schlieren'. Part 1 . Vertically oscillating cylinder. J. Fluid Mech. 390, 93-126.

Sutherland, B. R. \& Linden, P. F. 2002 Internal wave excitation by a vertically oscillating elliptical cylinder. Phys. Fluids 14, 721-731.

Tabaei, A., Akylas, T. R. \& Lamb, K. G. 2005 Nonlinear effects in reflecting and colliding internal wave beams. J. Fluid Mech. 526, 217-243.

Thomas, L. P., Marino, B. M. \& Dalziel, S. B. 2009 Synthetic schlieren: determination of the density gradient generated by internal waves propagating in a stratified fluid. J. Phys. Conf. Ser. 166, 012007.

Vlasenko, V., Stashchuk, N. \& Hutter, K. 2005 Baroclinic Tides: Theoretical Modeling and Observational Evidence. Cambridge University Press.

VoIsIn, B. 2003 Limit states of internal wave beams. J. Fluid Mech. 496, 243-293.

Voisin, B., Ermanyuk, E. V. \& FlóR, J.-B. 2011 Internal wave generation by oscillation of a sphere, with application to internal tides. J. Fluid Mech. 666, 308-357.

Zhang, H. P., King, B. \& Swinney, H. L. 2007 Experimental study of internal gravity waves generated by supercritical topography. Phys. Fluids 19, 096602.

Zhang, H. P., King, B. \& Swinney, H. L. 2008 Resonant generation of internal waves on a model continental slope. Phys. Rev. Lett. 100, 244504.

ZhANG, X. \& BoYer, D. L. 1993 Laboratory study of rotating, stratified, oscillatory flow over a seamount. J. Phys. Oceanogr. 23, 1122-1141. 